\title{
TOF-OFF: A method for determining focal positions in tightly focused free-electron laser experiments by measurement of ejected ions
}

\author{
B. Iwan ${ }^{\mathrm{a}}$, J. Andreasson ${ }^{\mathrm{a}}$, A. Andrejczuk ${ }^{\mathrm{b}}, \mathrm{E} . \mathrm{Abreu}^{\mathrm{c}}, \mathrm{M}$. Bergh $^{\mathrm{d}}$, C. \\ Caleman $^{\text {e }}$ A. J. Nelson ${ }^{f}$, S. Bajt ${ }^{g}$, J. Chalupsky ${ }^{\text {h }}$, H. N. Chapman ${ }^{\text {e, }}$, R. R. \\ Fäustlin $^{g}$, V. Hajkova ${ }^{\text {, }}$ P. A. Heimann ${ }^{j}$, B. Hjörvarssonk ${ }^{\text {, L. Luha }}$, D. \\ Klinger $^{\text {l, J. Krzywinskim }}{ }^{\mathrm{m}}$, B. Nagler ${ }^{\mathrm{m}}$, G. K. Pálsson ${ }^{\mathrm{k}}$, W. Singer ${ }^{\mathrm{g}}$, M. M. \\ Seibert $^{\mathrm{a}}$, R. Sobierajski ${ }^{\mathrm{l}, \mathrm{n}}$, S. Toleikis ${ }^{\mathrm{g}}$, T. Tschentscher ${ }^{\mathrm{o}}$, S. M. Vinko ${ }^{\mathrm{p}}, \mathrm{R}$. \\ W. Lee ${ }^{\mathrm{f}, \mathrm{m}}$, J. Hajdu ${ }^{\mathrm{a}}$, N. Tîmneanu ${ }^{\mathrm{a}, *}$ \\ ${ }^{a}$ Dept. of Cell and Molecular Biology, Uppsala University, Box 596, \\ SE-75124 Uppsala, Sweden \\ ${ }^{b}$ Faculty of Physics, University of Bialystok, Lipowa str. 41, PL-15424 Bialystok, Poland \\ ${ }^{c}$ Dept. of Physics, Boston University, 590 Commonwealth Avenue, Boston, \\ Massachusetts 02215, USA \\ ${ }^{d}$ Swedish Defence Research Agency, SE-16490, Stockholm, Sweden \\ ${ }^{e}$ Center for Free-Electron Laser Science, DESY, Notkestraße 85, \\ DE-22607 Hamburg, Germany \\ ${ }^{f}$ Lawrence Livermore National Laboratory, 7000 East Avenue, Livermore, \\ California 94550, USA \\ ${ }^{g}$ Deutsches Elektronen-Synchrotron DESY, Notkestraße 85, \\ DE-22607 Hamburg, Germany \\ ${ }^{h}$ Institute of Physics ASCR, Na Slovance 2, CZ-18221 Prague 8, Czech Republic \\ ${ }^{i}$ Dept. of Physics, University of Hamburg, Luruper Chausee 149, \\ DE-22761 Hamburg, Germany \\ ${ }^{j}$ Lawrence Berkeley National Laboratory, 1 Cyclotron Road, California 94720, USA \\ ${ }^{k}$ Dept. of Physics, Uppsala University, Box 530, SE-75121 Uppsala, Sweden \\ ${ }^{l}$ Inst. of Physics, Polish Academy of Sciences, AI. Lotnikow 32/46, \\ PL-02668 Warsaw, Poland \\ ${ }^{m}$ SLAC National Accelerator Laboratory, 2575 Sand Hill Road, Menlo Park, \\ California 94025, USA \\ ${ }^{n}$ FOM-Inst. for Plasma Physics Rijnhuizen, Edisonbaan 14, \\ NL-3430 Nieuwegein, The Netherlands \\ ${ }^{\circ}$ European XFEL GmbH, Albert-Einstein-Ring 19, DE-22761 Hamburg, Germany \\ ${ }^{p}$ Dept. of Physics, Clarendon Laboratory, University of Oxford, Parks Road, Oxford, \\ OX1 3PU, United Kingdom
}

\footnotetext{
${ }^{*}$ Corresponding author:

Email address: nicusor@xray.bmc.uu.se (N. Tîmneanu)
} 


\begin{abstract}
Pulse intensities greater than $10^{17}$ Watt $/ \mathrm{cm}^{2}$ were reached at the FLASH soft X-ray laser in Hamburg, Germany, using an off-axis parabolic mirror to focus 15 fs pulses of 5-70 $\mu \mathrm{J}$ energy at $13.5 \mathrm{~nm}$ wavelength to a micron-sized spot. We describe the interaction of such pulses with niobium and vanadium targets and their deuterides. The beam produced craters in the solid targets, and we measured the kinetic energy of ions ejected from these craters. Ions with several keV kinetic energy were observed from craters approaching 5 $\mu \mathrm{m}$ in depth when the sample was at best focus. We also observed the onset of saturation in both ion acceleration and ablation with pulse intensities exceeding $10^{16} \mathrm{~W} / \mathrm{cm}^{2}$, when the highest detected ion energies and the crater depths tend to saturate with increasing intensity.

A general difficulty in working with micron and sub-micron focusing optics is finding the exact focus of the beam inside a vacuum chamber. Based on our findings, we propose a method to measure the focal position to a resolution better than the Rayleigh length. The method is based on the correlation between the energies of ejected ions and the physical dimensions of the craters. We find that the focus position can be quickly determined without breaking vacuum, from the ion time-of-flight (TOF) data as the target is scanned through the expected focal region. Profile fitting employed to analyze the TOF data can extend resolution beyond the actual scanning step size.
\end{abstract}

Keywords: X-ray free-electron laser, FLASH, ion acceleration, time-of-flight ion spectrometry, ablation, crater formation, focus determination

\title{
1. Introduction
}

Recent developments in X-ray Free Electron Laser (XFEL) science have led to breakthrough results in several fields of research. With their extremely short, intense and coherent radiation pulses, X-ray lasers provide an unprecedented tool for the natural sciences. The FLASH Free Electron Laser in Hamburg [1] has made a mark in the soft X-ray regime, and the Linac Coherent Light Source (LCLS) in Stanford [2] is exploring the hard X-ray domain. New x-ray laser facilities are being built in Japan (SCSS, Spring-8 
Compact SASE Source) [3] and Italy (FERMI@Elletra, Free Electron Laser for Multidisciplinary Investigations) [4], and several similar light sources are being planned across the globe. These brilliant sources use X-rays to study physical processes relevant to a range of disciplines, from structural biology to plasma physics. In fact, these sources have already led to advances in coherent diffractive imaging [5] and femtosecond X-ray holography [6], cluster physics [7] as well as high-energy density physics $[8,9]$.

The FLASH Free Electron Laser in Hamburg is the first source that reached extreme conditions in laser-matter interactions in the soft X-ray regime, and showed that these interactions are very different from those found at optical frequencies [7-13]. Recent results from the LCLS hard X-ray laser $[14,15]$ confirm this picture, underlining the importance of understanding the interactions between high intensity X-ray radiation and materials. Controlling such interactions is fundamentally important to a range of disciplines including fusion research $[16,17]$. There, the aim is to maximize the deposited energy in the sample with the goal of achieving ion acceleration and ultimately obtaining fusion reactions. In a much different application, controlling radiation damage to molecular samples is essential for structural determination of single biomolecules [18], protein nanocrystals [19, 20], viruses [21] and biological cells [22, 23].

We have previously used theoretical modeling to describe the interaction of X-ray laser pulses with matter at high field strengths [22-24]. Furthermore, we have performed extensive computer simulations to explain experimental results obtained when using sub-micron focusing [25] of ultra-short pulses (15 fs) to reach intensities exceeding $10^{17} \mathrm{~W} / \mathrm{cm}^{2}$ on the surface of metallic samples: niobium, vanadium, and their deuterides [26, 27]. These simulations were performed using CRETIN [28], a multidimensional non - local thermodynamic equilibrium (non-LTE) plasma code for the study of population kinetics and radiation transfer. It incorporates established plasma models for low-density hot matter $[29,30]$ and follows atomic populations affected by X-ray laser-matter interactions. These simulations have been complemented with a self-similar isothermal fluid model [31] that describes how the ions at the plasma front are accelerated to energies exceeding the electron temperatures as the plasma expands into vacuum.

For a variety of experiments at FEL facilities, exact knowledge of the focus position of the laser is crucial since it allows one to exploit the full potential of the focused beam. Moreover, intensities on the sample can be calculated if the sample position relative to the beam focus is known. Traditionally, 


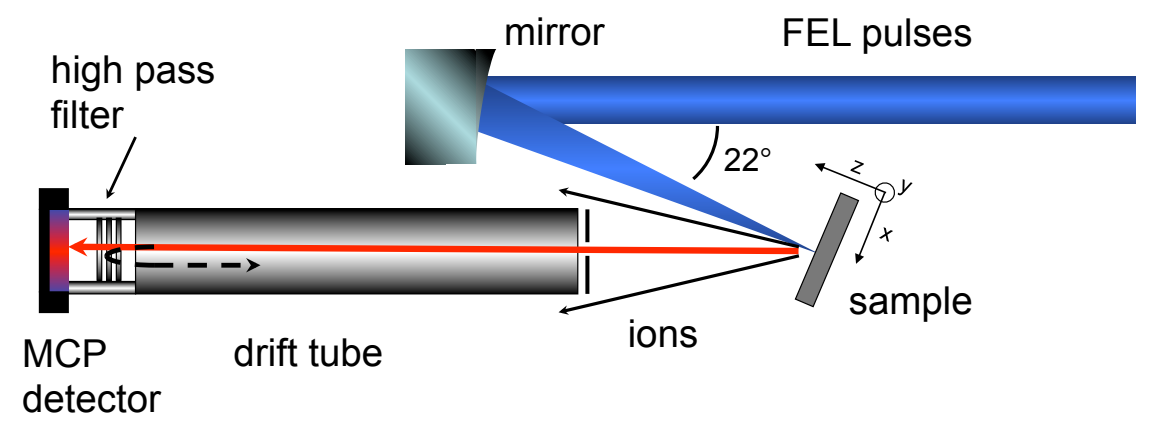

Figure 1: (Color online) Experimental setup used during experiments in April 2008 at FLASH. The incoming FEL beam is focused to a submicron spot size on the sample surface by a parabolic off-axis mirror. The sample can be moved in three directions $\mathrm{x}, \mathrm{y}$, and $\mathrm{z}$, where $\mathrm{z}$ is parallel to the FEL beam coming from the mirror. Ions ejected from the sample surface enter the drift tube of the TOF through a front-end aperture and are detected by the MCP after passing through a high pass filter grid. Whether an ion will pass the grid or is deflected depends on its kinetic energy and the grid voltage [26].

focus determination in a high intensity XFEL experiment is performed using offline analysis of imprints formed from attenuated shots on a suitable solid, like PMMA - Poly(methyl methacrylate) $[32,33]$. This process can be time consuming since it may require repeated access to the sample chamber and thus repeated venting cycles. We propose a complementary time-saving method based on in-situ focus determination. We call this method TOF$\mathrm{OFF}$ since it is based on the observation of a peak in the maximum kinetic energy of protons accelerated "off" the sample surface when the sample is at best focus.

\section{Experiments at FLASH}

FLASH was operated in single-bunch mode at $13.5 \mathrm{~nm}$ wavelength (corresponding photon energy $\mathrm{E}_{p h}=92 \mathrm{eV}$ ). The single pulses were about 15 fs long and had an average energy of $30 \mu \mathrm{J}$ with shot-to-shot variations ranging from 7 to $70 \mu \mathrm{J}$. Information about the pulse energy was obtained from an in-line gas monitor detector (GMD) [34], prior to arrival of the pulses at the interaction region. Sub-micron focusing of the FEL beam was achieved using an off-axis Mo/Si multilayer parabola [35] of focal length of $270 \mathrm{~mm}$, resulting in intensities that exceeded $10^{17} \mathrm{~W} / \mathrm{cm}^{2}$ in focus position $[9,25]$. 
The aspherical mirror had a reflectivity of $\mathrm{R}=67 \%$ and was mounted normal to the incoming FEL beam, off-set it by $22^{\circ}$ and focused it down to a sub-micron diameter (Fig. 1). Intensities were calculated based on the GMD information, pulse length and the calculated beam spot size on the sample surface. Initial measurements to determine the focal spot size were performed according to previously published methods [32] where the beam profile around best focus was determined by reference measurements with attenuated pulses on PMMA. This requires venting the chamber and an offline analysis of the imprints.

The measurements were performed on bulk niobium $(\mathrm{Nb})$ and vanadium (V). A subset of these has been doped with deuterium [36] resulting in deuterated niobium (NbD) and vanadium (VD) with a dopant/metal ratio of $0.680 \pm 0.002$ and $0.564 \pm 0.002$, respectively. The samples were polished prior to mounting into the sample holder. The single crystal $\mathrm{Nb}(10 \times 10 \times 3 \mathrm{~mm})$ was electropolished, whereas the polycrystalline Nb, V and VD $(12 \times 12 \times 1$ $\mathrm{mm}$ ) sample plates were mechanically polished. Prior to our investigation in vacuum, all the samples were handled at atmospheric pressure and thus have surface contamination from water and air diffusion into the outermost atomic layers, prominently hydrogen $(\mathrm{H})$, oxygen $(\mathrm{O})$ and carbon $(\mathrm{C})$.

Finally, the samples were mounted normal to the axis of the focused FEL beam, as shown in Fig. 1. This setup allowed for motion in directions parallel and perpendicular to the focused beam (labelled as $\mathrm{x}, \mathrm{y}$, and $\mathrm{z}$, with $\mathrm{z}$ being the beam direction). Thus, the intensity on the sample surface could easily be varied by moving the sample in and out of focus along the beam in the $\mathrm{z}$ direction.

A time of flight (TOF) spectrometer [37] was mounted parallel to the incoming FEL beam opposite the sample holder, see Fig. 1. Ion detection was accomplished with a multichannel plate (MCP) detector at the end of a grounded drift tube approximately $600 \mathrm{~mm}$ away from the interaction region (Fig. 1). The narrow acceptance angle of the TOF front-end aperture limited the ion transmission to the detector. Additional high pass (HP) filter grids in front of the MCP allowed for selection of a specific ion energy range. Only ions ejected towards the TOF and with kinetic energies above a certain threshold were detected. For example, for a grid voltage of $2 \mathrm{kV}$ only ions with a kinetic energy above $\mathrm{q} \times 2 \mathrm{keV}$, where $\mathrm{q}$ is the charge state of the ion, passed the HP filter and ions with lower energy were deflected. For moderate grid voltages, i.e. up to $2.5 \mathrm{kV}$, we observed multiply charged metal ions from the bulk sample as well as $\mathrm{H}^{+}, \mathrm{C}^{+}$and $\mathrm{O}^{+}$from surface 


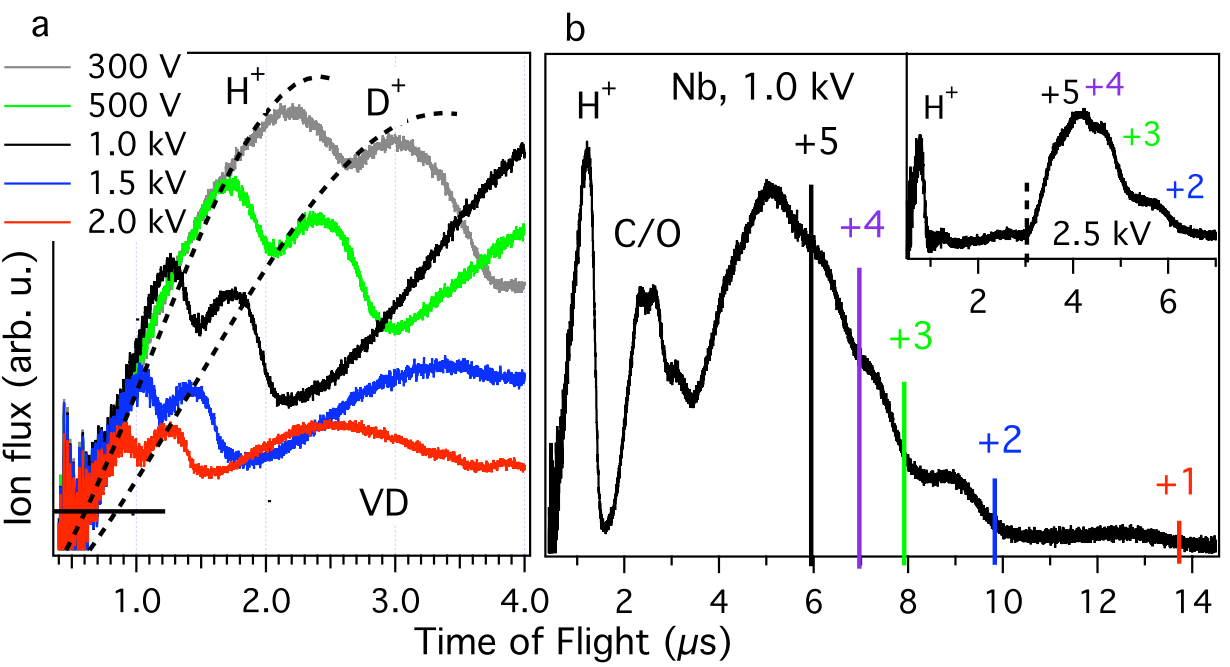

C

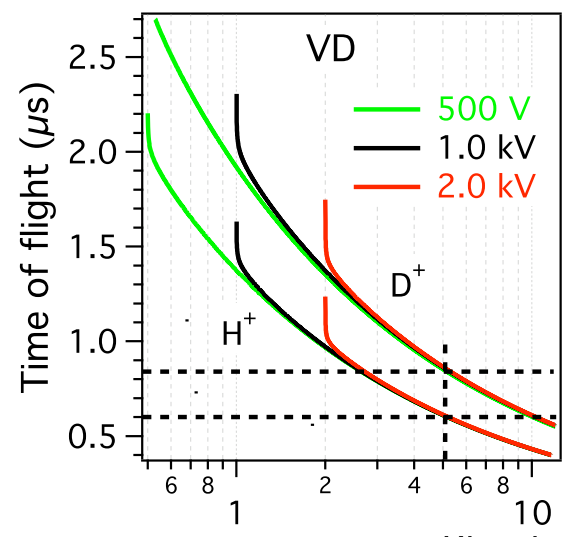

d

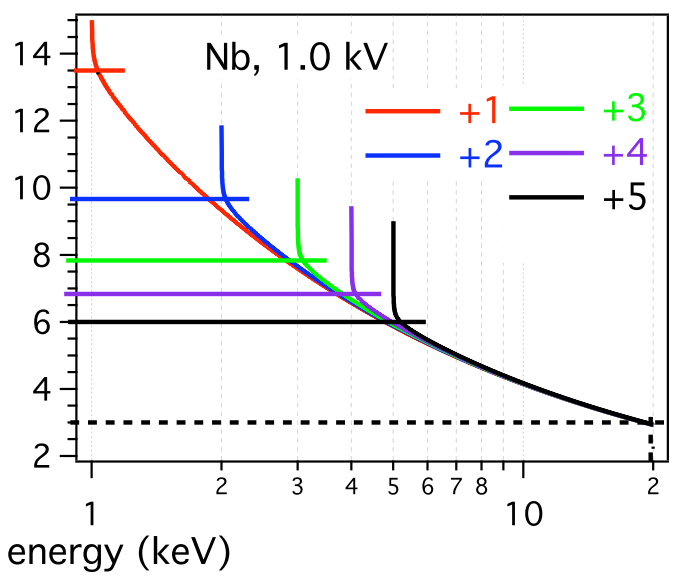

Figure 2: (Color online) (a) TOF spectra for singly charged light ions from $\mathrm{NbD}$ with varying high pass filter voltages ranging from $0.3 \mathrm{kV}$ to $2 \mathrm{kV}\left(\mathrm{H}^{+}\right.$from surface contamination, $\mathrm{D}^{+}$from inside the metal structure). (b) TOF spectra for multiply charged metal ions $(\mathrm{Nb})$ with a grid voltage of $1 \mathrm{kV}$. The insert shows the corresponding spectrum at a grid voltage of $2.5 \mathrm{kV}$. In (c) and (d) Simion simulations of light ions and metal ions are presented. See text for further details. 
contaminations. Higher voltages resulted in a clean spectrum containing only signal from highly energetic protons from the surface and metal ions, without traces of surface contamination from low energetic $\mathrm{O}^{+}$and $\mathrm{C}^{+}$, see inset in Fig. 2 (b). The suppression of the peaks from $\mathrm{O}^{+}$and $\mathrm{C}^{+}$ions developed with increasing grid voltage showing that these ions did not reach energies above $2.5 \mathrm{keV}$. However, hydrogen, also from surface impurities, could reach much higher energies; a fact that is the basis for experiments with laserdriven proton acceleration [38]. Since no other acceleration fields were used we can directly correlate the detected ion time of flights with the conditions in the interaction region and the subsequent ion acceleration processes. For deuterated samples, where the deuterium has diffused out of the surface layer by the time of the experiment, a significant TOF signal due to deuterium ions $\left(\mathrm{D}^{+}\right)$could only be recorded while irradiating an already exposed section of the sample, thus reaching below the depleted surface layer. This selectivity in deuterium observation and the different ways in which the above mentioned carbon and oxygen signals responds to increased grid voltages represent two ways in which bulk and surface effects in the ion acceleration and crater formation processes can be separated.

Assignment of the ion time of flight signals was done using Simion [39], an ion time-of-flight simulation software. From information about the voltages and experimental geometry used for the measurements, the program calculates the resulting electric fields and simulates ion trajectories for a choice of ions ( $\mathrm{Nb}, \mathrm{V}, \mathrm{H}, \mathrm{D}, \mathrm{O}$ and $\mathrm{C})$ and charge states $(+1$ to +8$)$. These trajectories will determine the time of flights, kinetic energies and ion velocities that in turn can be mapped on to the experimental data to enable a correct time-to-energy transformation and mass over charge (m/q) assignment, as shown in Fig. 2. The simulated flight times for ions show cutoffs that correspond well with the cutoffs in the experimental TOF traces and allow for a correct assignment of charge states and ion mass as well as kinetic energy. The horizontal dotted lines in Fig. 2 (c) and (d) correspond to the highest detected energies with $95 \%$ confidence level, shown in Fig. 2 (a) and the inset in (b).

The main sources of error when calculating the intensity on the sample are: 1) uncertainties in the GMD data of $\sim 10 \%[12]$; 2) a decreased reflectivity in the parabolic mirror observed during seven days of measurement (from $67 \%$ to $50 \%$ ) [25]; and, 3) uncertainties in the $\mathrm{z}$ position of the sample relative to the mirror $( \pm 0.05 \mathrm{~mm})$ due to limitations in the accuracy of the sample stage position. 


\section{In-situ focus determination with the ion TOF spectrometer}

Fig. 3 shows the drift ion TOF spectra for $\mathrm{H}^{+}$ions originating from surface impurities as a function of the time of flight. We label the sample position $\mathrm{z}=0.0$ as the position that yields the highest observed proton energy and tentatively assign this position to the location of the focal plane of the mirror. Hydrogen has been detected from all four materials and a strong dependency on the sample position (z-position) relative to the FEL focus is seen in the observed proton flight times (and thus, kinetic energies). The highest observable proton energy is defined as the energy corresponding to the flight time where the proton signal is detected above the background with a confidence level of $95 \%$. Moving the sample $0.2 \mathrm{~mm}$ away from $\mathrm{z}=0.0$ results in a decrease in the ion yield and longer flight times for the most energetic protons. This distinctly observed maximum proton energy leads us to propose this TOF-OFF method as a convenient method for in-situ determination of best focus.

Although the present measurements were made moving the sample surface with a large step size of $0.2 \mathrm{~mm}$, the TOF-OFF method is able to find the position of the focal plane with a far better accuracy. The slope of the maximum measured proton energy in the range $\mathrm{z}=-0.6 \mathrm{~mm}$ to $\mathrm{z}=0.0 \mathrm{~mm}$ in Fig. 4 shows that a $50 \mu \mathrm{m}$ movement of the sample corresponds to a change in the observed energy of about $200 \mathrm{eV}$. The Simion simulations show this difference in energy can correspond to a shift of $200 \mathrm{~ns}$ in the onset of the proton peak, which is clearly observable in the TOF spectra (Fig. 3). Figure 4 also presents the intensities corresponding to the sample position relative to best focus, which are calculated using the size of the focal spot previously determined using attenuated pulses on PMMA [25]. The logarithmic scale on the upper axis indicates that the kinetic energies experience saturation when intensities exceed $10^{16} \mathrm{~W} / \mathrm{cm}^{2}$.

Assuming symmetry of the laser beam on both sides of the focal plane, the exact location of the focal plane can be determined by a peak fitting procedure. The experimental data for $\mathrm{V}, \mathrm{VD}$ and $\mathrm{NbD}$ in Fig. 4 is fit to a Lorentzian peak profile of the form $\frac{a}{b+\left(z-z_{0}\right)^{2}}$. This form is suitable as a first approximation to describe the dependence of the intensity with the focal area, which is proportional to $z^{2}$. The location of the focal plane $z_{0}$ relative to the positions where we measured the highest proton energies is given by the function maxima. We see that for $\mathrm{NbD}$ the initial assignment of the $\mathrm{z}=0.0$ position to the focal plane of the mirror is verified, as confirmed by 

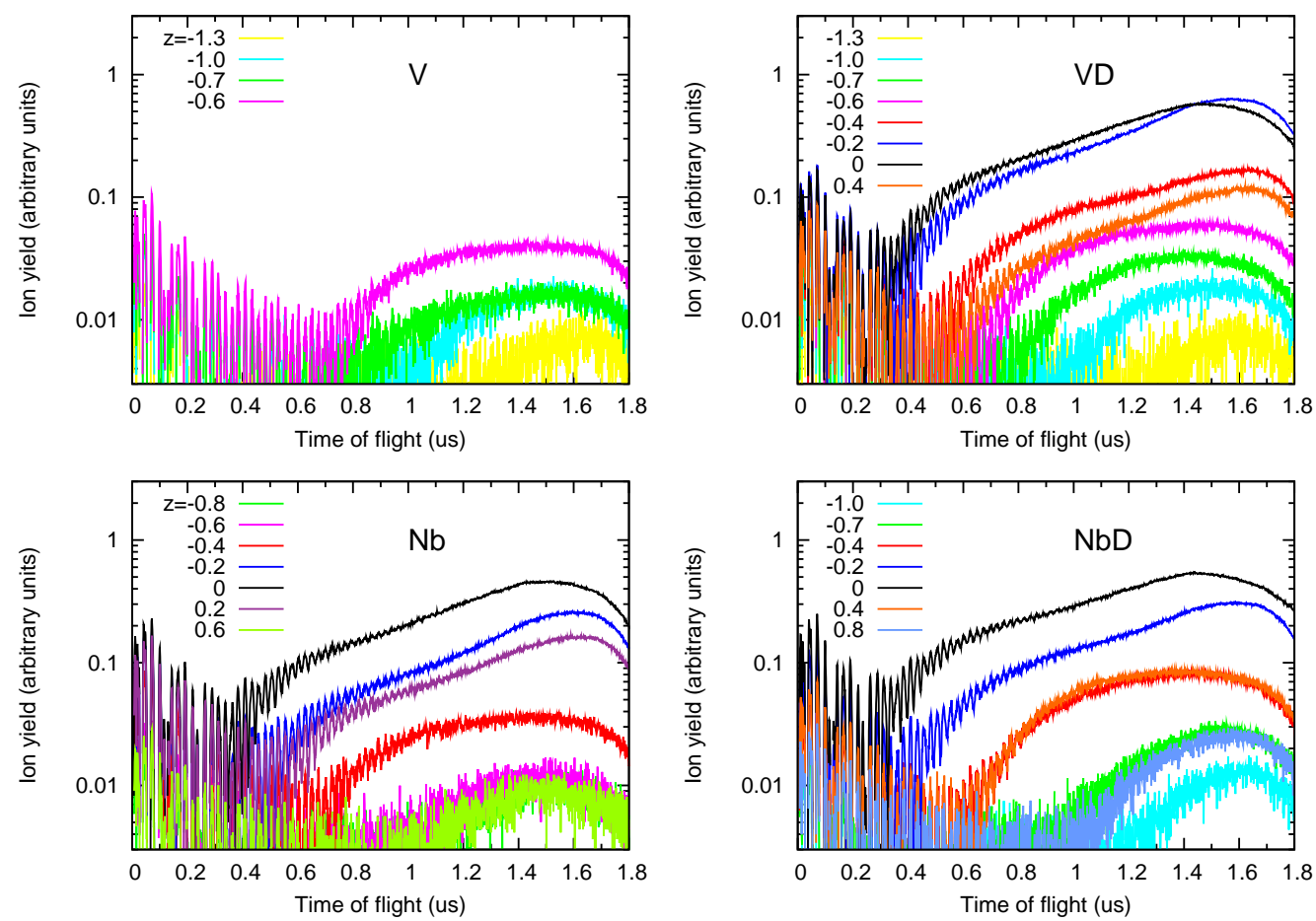

Figure 3: (Color online) Acceleration of protons from a solid surface. Shown is the hydrogen $(\mathrm{m} / \mathrm{q}=1)$ signal from surface impurities on all four samples used in the experiment as a function of flight time. The position $\mathrm{z}=0.0 \mathrm{~mm}$ is the position where the highest proton energies were observed and is considered to be closest to the focal plane of the mirror. The spectra for hydrogen show the same behavior in each material. Shorter time of flights are observed when approaching the best focus $(\mathrm{z}=0.0 \mathrm{~mm})$ position, resulting in higher energies of the protons. In addition, going away from the position of best focus results in a decrease in the ion yield. The average pulse energy of the X-ray pulse is $30 \mu \mathrm{J}$ and the applied grid voltage is $500 \mathrm{~V}$. Ion drift TOF traces were taken at different sample locations and each trace is an average of at least 30 measurements. 

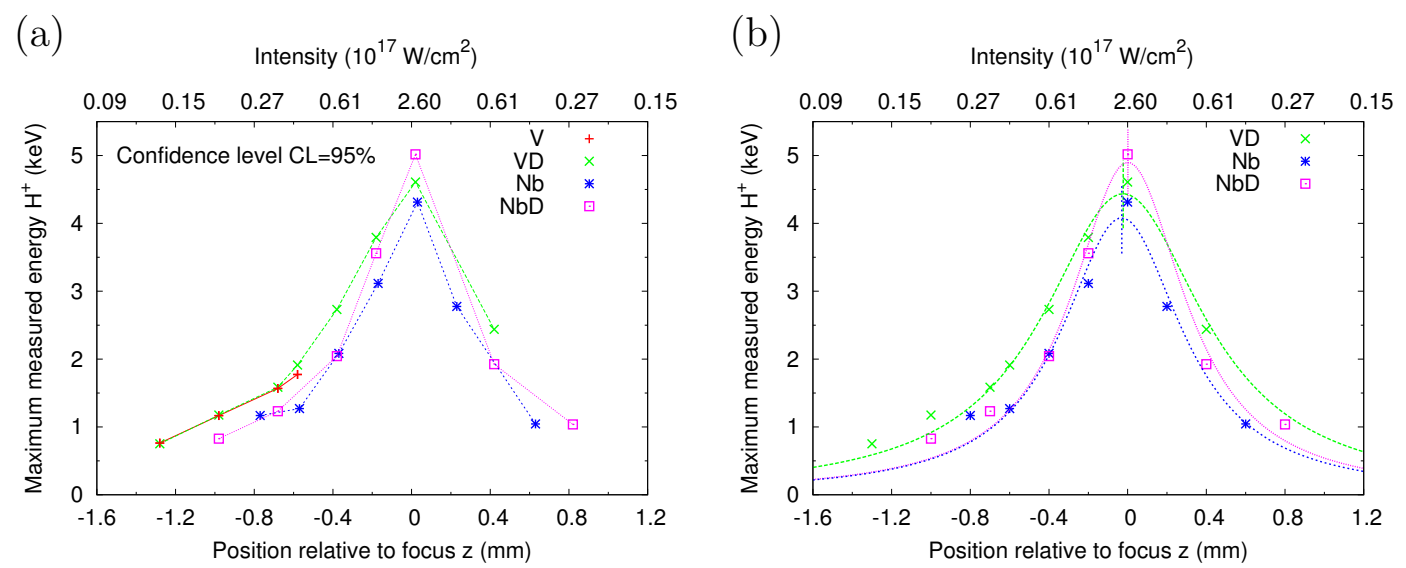

Figure 4: (Color online) (a) Maximum measured kinetic energy of hydrogen for all four samples as a function of the position relative to best focus $\mathrm{z}=0.0$ where the highest kinetic energies are detected. Moving the sample away from this position results in a steady decline. The experiments have been performed over a larger range around the focus position for all samples, except $\mathrm{V}$. The confidence level for detection of signal above the background is at $95 \%$. (b) Profile fitting to the data with a Lorentzian function allows an exact determination of the focal position at the peak of the fitted curves (vertical lines). The $\mathrm{z}=0.0$ position is well aligned with the focal plane for $\mathrm{NbD}$ and is about $30 \mu \mathrm{m}$ off for $\mathrm{VD}$ and $\mathrm{Nb}$.

the proton traces observed for NbD at $\pm 0.4 \mathrm{~mm}$ which show a high degree of overlap (Fig. 3). For VD and $\mathrm{Nb}$ the peak-fitting procedure locates the focal plane $30 \mu \mathrm{m}$ away from the $\mathrm{z}=0.0$ position. This slight mismatch is also observed in Fig. 3 where the ion yields from VD at $\mathrm{z}= \pm 0.4 \mathrm{~mm}$ and $\mathrm{Nb}$ at $\mathrm{z}= \pm 0.2 \mathrm{~mm}$ are slightly off. We conclude that the TOF-OFF method is capable of determining the location of the focal plane with an accuracy better than $30 \mu \mathrm{m}$ and note that this accuracy is well within the Rayleigh length of the mirror which is estimated to $\pm 75 \mu \mathrm{m}$. It is interesting to note that the present method captures the slight misalignment of $30 \mu \mathrm{m}$ between the surface of different samples although they are mounted in the same sample holder and should ideally be in the same plane. This implies that a further advantage of the TOFF-OFF method is that the determination of the sample location relative to the focal plane of the mirror is made simultaneously with the measurement. This avoids uncertainties related to the limitations in the reproducibility of the sample environment, e.g. sample mounting of motor accuracy, going from a sample used for focus determination to another sample of scientific significance. 
It has been previously shown that intensities approaching $10^{17} \mathrm{~W} / \mathrm{cm}^{2}$ are accompanied by a saturation in the maximum proton kinetic energy [26]. This can be traced back to saturation in surface absorption due to rapid inner shell processes and results in a decline in proton acceleration. It is seen from Fig. 4 that the TOF-OFF approach can be used for intensities up to, and above, $10^{17} \mathrm{~W} / \mathrm{cm}^{2}$ despite the onset of saturation in proton kinetic energies. However, the best resolution is possible in the intensity range where there is a high rate of increase of the maximum observable proton energy with intensity. Using attenuation on the XFEL beam the TOF-OFF method should be useful also when the maximum intensity is associated with significant saturation. In addition to the proton signal the TOF spectra show highly energetic metal ions. For these ions, charge states of up to +5 have been clearly distinguished with flight times of $3 \mu \mathrm{s}$, corresponding to a kinetic ion energy of $20 \mathrm{keV}$ (Fig. 2 (b)). The occurrence of highly charged and very energetic ions is known from previous experiments on semiconductors with extreme ultraviolet pulses [40].

Confirmation for the best focus position obtained from the TOF-OFF measurements has been ascertained by studying the craters formed in the sample at different sample locations relative to the position that gave the highest ion TOF signal. The craters were characterized by scanning electron microscopy (SEM) seen in Fig. 5 and measurements of crater depths were carried out with tapping mode atomic force microscopy (AFM) shown in Fig. 6 and Figures in Ref. [26]. The craters formed on the sample surface show a similar strong dependency on the z-position of the sample, which corresponds to the intensity, as the observed maximum proton energy. Figure 5 shows SEM images of craters formed by single FEL pulses with pulse energies close to the average $30 \mu \mathrm{J}$ for different sample positions relative to the location of best focus. For in-focus position $(\mathrm{z}=0.0 \mathrm{~mm})$ deep and narrow craters were observed with a diameter of about $5 \mu \mathrm{m}$ and depths exceeding $3 \mu \mathrm{m}$ (Fig. 5 (f)). When moving the sample away from the in-focus position craters tend to become shallower and the ablated area increases resulting in imprints with a diameter larger than $10 \mu \mathrm{m}$ on the surface (Fig. 5 (a)-(e), $(\mathrm{g}),(\mathrm{h}))$. It has been observed that changes in pulse energy at the same sample position may result in significant differences in crater formation [26]. This is likely correlated to an increase in transport of energy out the sides of the craters when deeper craters are formed.

The correspondence of the position of best focus as determined by the ion TOF measurements and crater SEM imaging is further strengthened by an 


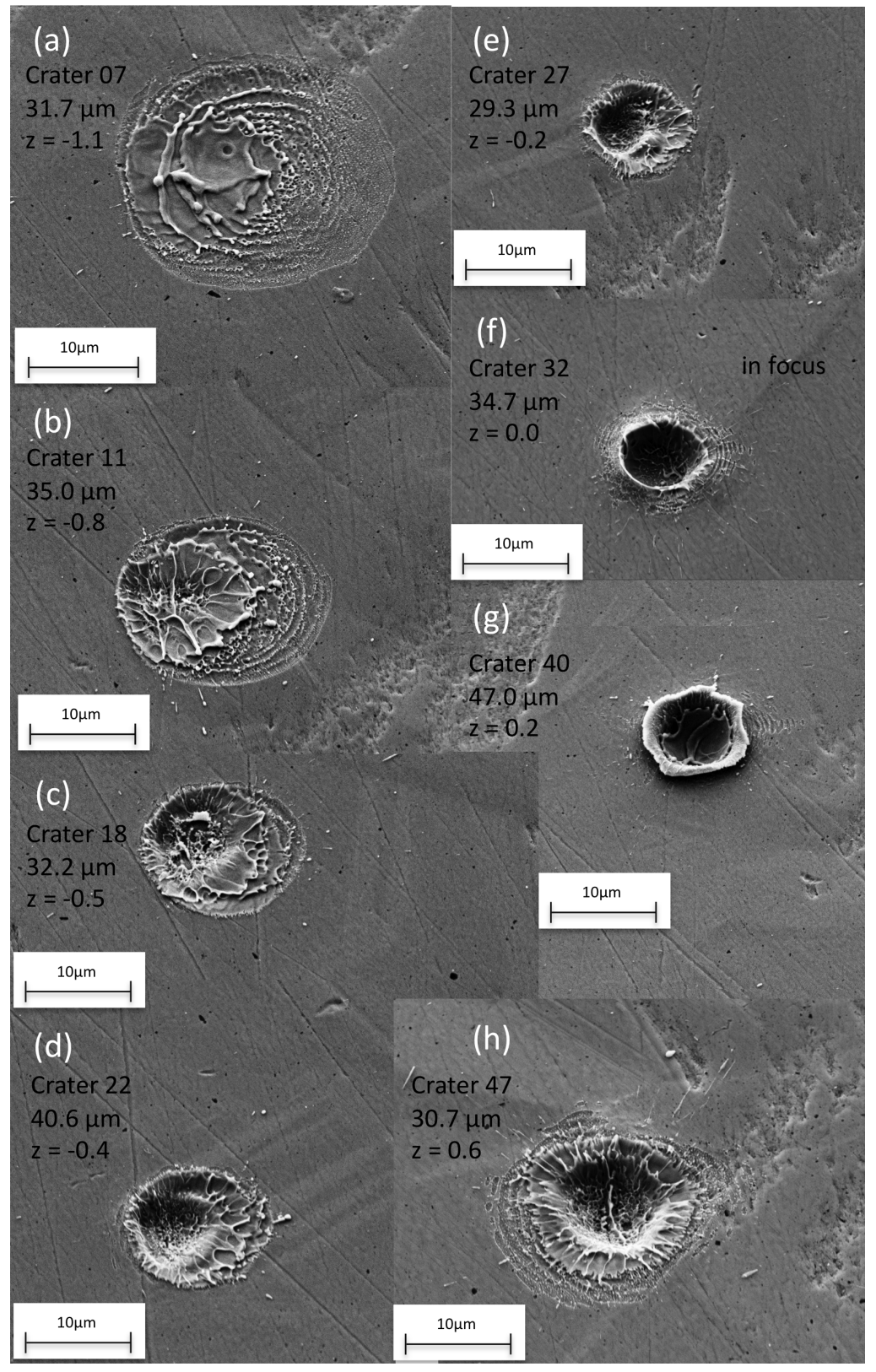

Figure 5: Crater formation for different relative $\mathrm{z}$ position as measured with SEM on deuterated vanadium (VD). (a) - (d) For out-of-focus positions wide and shallow imprints can be seen on the sample surface. Clearly visible is a fringe pattern which is the result of the FEL beam being scattered by an upstream aperture [26]. (e) - (g) Close to and at best focus $(\mathrm{z}=0.0 \mathrm{~mm})$ the craters become deeper and more pronounced until a maximum depth is reached. (h) Passing the best focus leads to larger and shallower craters. See text for further details. 
analysis of the observed crater depths with tapping mode atomic force microscopy (AFM). Fig. 6 displays AFM measurements of the depths of a subset of craters formed in $\mathrm{NbD}$ when the sample surface is located at different distances from best focus $(\mathrm{z}=-0.8,-0.4,-0.2,0.0$ and $0.2 \mathrm{~mm}$ ) as determined by the TOF-OFF method (for a full crater analysis, see [26]). The dependence with incident intensity is also shown in the upper scale, where the intensity has been calculated under the assumption that the focal spot size was sub-micron at the best focus position, as determined previously [25]. Our measurements show that the deepest craters are formed when the sample surface is near the best focus position determined by ion TOF spectrometry. It is also seen that it is not possible to verify the position of best focus with the crater data obtained at the highest pulse energies due to saturation in the crater depth. However, for pulses with low to moderate intensities (20-25 $\mu \mathrm{J})$, a post experiment analysis of the craters with AFM confirms the range of the best focus derived from the maximum observable proton kinetic energy through the TOF-OFF method (Fig. 5 and 6). In cases where saturation is observed attenuated pulses can be used to avoid misleading interpretation of the data.

We have experimental evidence that there exists a strong correlation between sample position relative to the laser focus, intensity, proton kinetic energies and crater appearance. Acceleration of protons from surface contamination can reach $\mathrm{keV}$ energies and the highest measurable proton energies will be from samples whose surfaces are irradiated while being at best focus (Fig. 4). The position of best focus found by an analysis of the TOF-OFF data can be verified by a subsequent analysis of the craters formed in the samples. By exploiting this strong correlation between crater formation and intensities as well as maximum proton energies, one can determine the focal plane of the laser and hence the best sample position with a quick analysis of the hydrogen peak in the measured TOF spectra. This can be performed online with the sample remaining in the experimental chamber.

\section{Summary}

We propose a novel method for in-situ focus determination for high intensity soft X-ray and X-ray experiments. The TOF-OFF method is based on the observation that the maximum kinetic energy obtained by protons accelerated off the sample surface by the focused FEL beam is strongly dependent on photon intensity and reaches a maximum when the sample is 
(a)
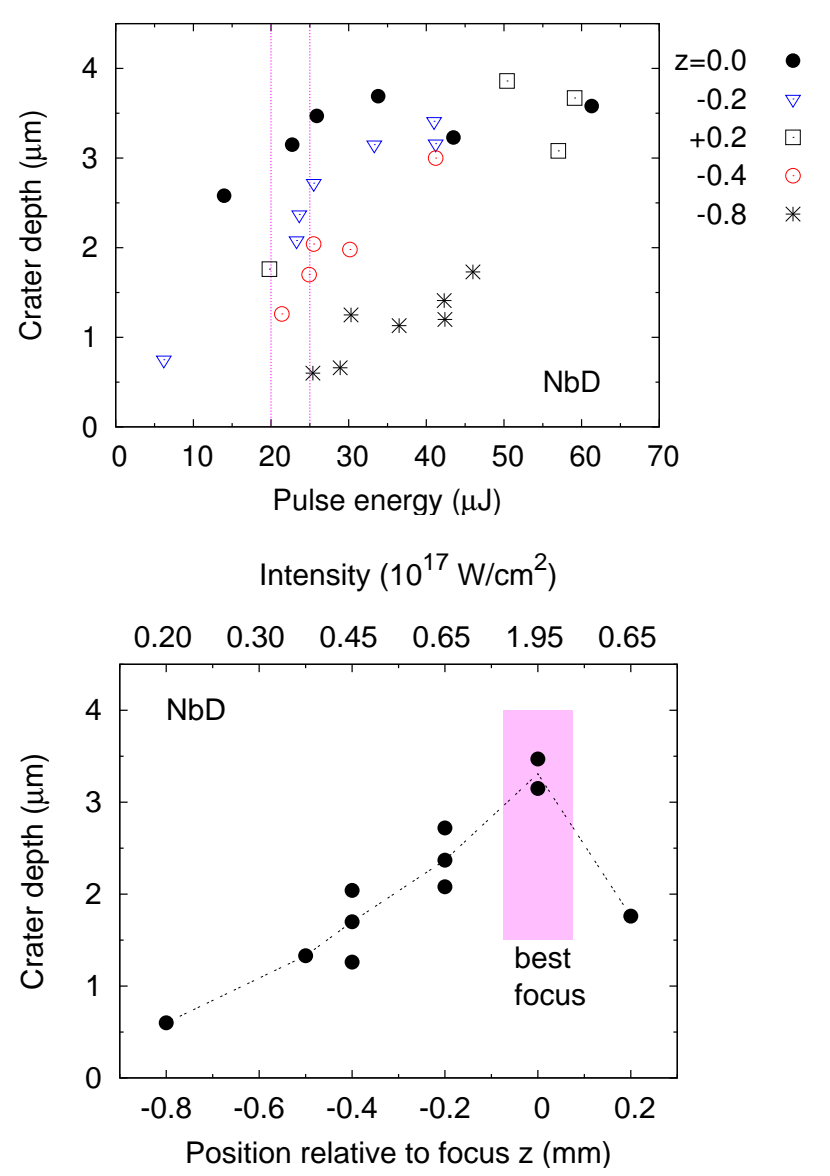

Figure 6: (Color online) (a) Crater depth in $\mathrm{NbD}$ as a function of pulse energy for different sample locations with respect to the position of best focus determined by the TOF-OFF method $(\mathrm{z}=-0.8,-0.4-0.2,0.0$ and $0.2 \mathrm{~mm})$. The measured crater depth increases with increasing pulse energy for each sample position and when the sample is moved to the position of best focus as determined by the TOF-OFF method. (b) Crater depth as a function of sample position relative to the best focus for craters formed at lower pulse energies (20 to $25 \mu \mathrm{J}$ ). For intensities below $10^{17} \mathrm{~W} / \mathrm{cm}^{2}$ the crater depth analysis confirms the position of best focus. The shaded region at best focus marks the size of the Rayleigh length $( \pm 75 \mu \mathrm{m})$. 
moved into the position of best focus. Subsequent SEM and AFM analysis of the craters formed in the sample surfaces verify that the position of the best focus obtained with the TOF-OFF method indeed coincides with the focal plane of the optics determined to an accuracy similar to the Rayleigh length. This method is complementary to the methods traditionally used but does not require the removal of the sample from the chamber for off-line analysis.

\section{Acknowledgements}

We thank the staff at FLASH for the tremendous help during the experiment. This work was supported by the following agencies: The Swedish Research Council through a Centre of Excellence Award to J.H., the Virtual Institute Program of the Helmoltz Association (VH-VI-302), the Helmholtz Association through the Center for Free Electron Laser Research, the DFG Cluster of Excellence at the Munich Centre for Advanced Photonics, the Portuguese Science and Technology Foundation, the Czech Ministry of Education (LC510, LC528, ME10046 and LA08024) and Academy of Sciences (AV0Z10100523, IAAX00100903, and KAN300100702), and the MSHE of Poland, SPB nr.DESY/68/2007. Computations were performed on UPPMAX under project p2009018. SEM measurements were performed at the Microscopy and Microanalysis Group, Chalmers University of Technology. Access to FLASH was supported by the European Union under contract RII3-CT-2004-506008 (IA-SFS).

\section{References}

[1] V. Ayvazyan, N. Baboi, J. Bähr, et al., First operation of a free-electron laser generating GW power radiation at $32 \mathrm{~nm}$ wavelength, Eur. Phys. J. D 37 (2006) 297.

[2] P. Emma, R. Akre, J. Arthur, et al., First lasing and operation of an angstrom-wavelength free-electron laser, Nat. Photonics 4 (2010) 641647.

[3] T. Shintake, H. Tanaka, T. Hara, et al., Stable operation of a selfamplified spontaneous-emission free-electron laser in the extremely ultraviolet region, Phys. Rev. ST Accel. Beams 12 (2009) 070701. 
[4] FERMI@Elettra Conceptual Design Report, available at http://www.elettra.trieste.it/FERMI

[5] H. N. Chapman, A. Barty, M. J. Bogan, et al., Femtosecond diffractive imaging with a soft-X-ray free-electron laser, Nature Phys. 2 (2006) 839.

[6] H. N. Chapman, S. P. Hau-Riege, M. J. Bogan, et al., Femtosecond time-delay X-ray holography, Nature 448 (2007) 676-679.

[7] C. Bostedt, H. Thomas, M. Hoener, et al., Multistep Ionization of Argon Clusters in Intense Femtosecond Extreme Ultraviolet Pulses, Phys, Rev. Lett. 100 (2008) 133401.

[8] J. Chalupský, L. Juha, V. Hájková, et al., Non-thermal desorption/ablation of molecular solids induced by ultra-short soft X-ray pulses, Optics Express 17 (2009) 208.

[9] B. Nagler, U. Zastrau, R. R. Fäustlin, et al., Turning solid aluminium transparent by intense soft X-ray photoionization, Nature Phys. 5 (2009) 693.

[10] A. A. Sorokin, S. V. Bobashev, T. Feigl, et al., Phys. Rev. Lett 99 (2007) 213002 .

[11] H. Wabnitz, L. Bittner, A. R. B. de Castro, et al., Nature 420 (2002) 482 .

[12] S. P. Hau-Riege, R. A. London and H. N. Chapman, Phys. Rev. E 76 (2007) 046403.

[13] S. M. Vinko, U. Zastrau, S. Mazevet, et al., Phys. Rev. Lett. 104 (2010) 225001.

[14] J. P. Cryan, J. M. Glownia, J. Andreasson, et al., Phys. Rev. Lett. 105 (2010) 083004.

[15] L. Young, E. P. Kanter, B. Krssig, et al., Nature 466 (2010) 56.

[16] T. Ditmire, J. Zweiback, V. P. Yanovsky, et al., Nature 398 (1999) 489.

[17] M. Roth, T. E. Cowan, M. H. Key, et al., Phys. Rev. Lett. 86 (2001) 436. 
[18] R. Neutze, R. Wouts, D. van der Spoel, et al., Nature 406 (2000) 752.

[19] H. N. Chapman, P. Fromme, A. Barty, et al., Nature 470 (2011) 73-81.

[20] C. Caleman, G. Huldt, F. R. N. C. Maia, et al., ACS Nano 5 (2011) $139-146$.

[21] M. M. Seibert, T. Ekeberg, F. R. N. C. Maja, et al., Nature 470 (2011) $78-86$.

[22] M. Bergh, G. Huldt, N. Tîmneanu, et al., Q. Rev. Biophys. 41 (2008) 181.

[23] M. M. Seibert, S. Boutet, M. Svenda, et al., J. Phys. B - At. Mol. Opt. Phys. 43 (2010) 194015.

[24] M. Bergh, N. Tîmneanu, S. P. Hau-Riege, et al., Phys. Rev. E 77 (2008) 026404 .

[25] A. J. Nelson, S. Toleikis, H. Chapman, et al., Opt. Express 17 (2009) 18271.

[26] J. Andreasson, B. Iwan, A. Andrejczuk, et al., Phys. Rev. E 83 (2011) 016403.

[27] B. Iwan, J. Andreasson, A. Andrejczuk, et al., Proc. of SPIE (2011) in press.

[28] H. A. Scott, S.B. Hansen, High Energy Density Phys. 6 (2009) 39.

[29] M. Fajardo, P. Zeitoun and J.-C. Gauthier, Eur. J. Phys. D 29 (2004) 69.

[30] K. Eidmann, J. Meyer-ter-Vehn, T. Schlegel, et al., Phys. Rev. E 62 (2000) 1202.

[31] P. Mora, Phys. Rev. Lett. 90 (2003) 185002.

[32] J. Chalupský, L. Juha, J. Kuba, et al., Opt. Express 15 (2007) 6036.

[33] J. Chalupský, P. Bohacek, V. Hajkova, et al., Nucl. Instrum. Meth. A 631 (2011) 130-133. 
[34] K. Tiedtke, A. Azima, N. von Bargen, et al., New J. Phys. 11 (2009) 023029 .

[35] S. Bajt, H. N. Chapman, A. J. Nelson, et al., Proc. of SPIE 7631 (2009) $76310 \mathrm{~J}$.

[36] G. K. Pálsson,A. R. Rennie and B. Hjörvarsson, Phys. Rev. B 78 (2008) 104118.

[37] R. Sobierajski, J. Krzywinski, A. Andrejczuk, et al, Rev. Sci. Instrum. 76 (2005) 013909.

[38] J. Fuchs, P. Antici, E. dHumères, et al., Nature Phys. 2 (2006) 48.

[39] D. Dahl, Int. J. Mass Spectrom. 200 (2000) 3.

[40] J. Krzywinski, R. Sobierajski, M. Jurek, et al., J. Appl. Phys. 101 (2007) 043107. 